\title{
Enhancing the Response of Alkyl Methylphosphonic Acids in Negative Electrospray Ionization Liquid Chromatography Tandem Mass Spectrometry by Post-Column Addition of Organic Solvents
}

\author{
Douglas B. Mawhinney, Rayman D. Stanelle, ${ }^{+}$Elizabeth I. Hamelin, ${ }^{+}$ \\ and Robert J. Kobelski \\ U.S. Centers for Disease Control and Prevention, National Center for Environmental Health, Division of \\ Laboratory Sciences, Emergency Response and Air Toxicants, Atlanta, Georgia, USA
}

A method to enhance the signal intensity and signal-to-noise of several alkyl methylphosphonic acids in negative electrospray ionization liquid chromatography tandem mass spectrometry (ESI LC-MS/MS) is presented. This class of compound represents the initial metabolites and environmental degradants of the nerve agents: VX, rVX (Russian VX), GB (Sarin), GF (Cyclosarin), and GD (Soman). Compared with the post-column addition of the mobile phase, the post-column addition of aprotic solvents and longer chain alcohols enhance the signal intensity and signal-to-noise ratio $(\mathrm{S} / \mathrm{N})$ of the chromatographic peaks by factors of up to 60 and 19, respectively. The post-column addition of water, methanol, and ethanol resulted in little or no relative signal enhancement. It is proposed that the post-column addition of these solvents do not result in the same enhancements due to stabilization of analyte solvation through hydrogen bonding. (J Am Soc Mass Spectrom 2007, 18, 1821-1826) (C) 2007 American Society for Mass Spectrometry

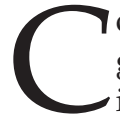
oncern over the release of nerve agents has been growing recently due to terrorism, the processes involved in chemical weapons demilitarization, and the continued existence of chemical stockpiles [1]. In response to this concern, there is interest in detecting these compounds and their primary hydrolysis products, alkyl methylphosphonic acids, at increasingly lower concentrations. The public health sector is interested in detecting trace amounts of these compounds as markers for nerve agent exposure because they also represent the primary metabolites of the nerve agents. The current methods employed for this application typically use liquid chromatography coupled to tandem mass spectrometry for detection. The most recent public health related method has limits of detection (LOD) in the range of 0.8 to $6 \mathrm{pg}$ on-column [2], and there is interest in increasing the sensitivity of the analysis. To that end, we have been investigating ways to optimize

Address reprint requests to Dr. D. B. Mawhinney, U.S. Centers for Disease Control and Prevention, National Center for Environmental Health, Division of Laboratory Sciences, Emergency Response and Air Toxicants, 4770 Buford Highway NE, Mailstop F-44, Atlanta, Georgia 30341, USA. E-mail: dbmawhinney@hotmail.com

* Current address: Southern Nevada Water Authority, 1350 Richard Bunker Avenue, Henderson, Nevada 89015, USA

+ Battelle Memorial Institute, under contract with the U.S. Centers for Disease Control and Prevention, National Center for Environmental Health. negative electrospray ionization conditions to increase the number of analyte ions formed.

The generation of gas-phase ions by electrospray ionization depends heavily upon the composition of the droplets formed at the beginning of the process. Much work has focused on analyte response suppression due to the deleterious effect on sensitivity and reproducibility, as well as to help understand the dynamics of the electrospray process [3-9]. Conversely, analyte response has been increased through lower flow rates [10] and the addition of modifiers to the electrosprayed solvent [6, 11], as well as through improved sample clean-up strategies [6]. The ability to control suppression and enhancement is the ultimate goal, whether it is to increase the quality of quantitative data, reduce background contributions, or increase the sensitivity to specific compounds.

Some basic concepts of electrospray ionization with links to signal enhancement have been discussed in the literature, with most of the studies based on infusion mass spectrometry experiments. Tang and Kebarle [12, 13] proposed a model, which was further developed by Enke [14], where instrumental response is based on the rate of delivery of the analyte ion versus the background electrolyte into the surface excess charge layer from the interior charge-balanced layer. As an extension of this work, Constantopoulos et al. proposed the 
formation of a multilayer droplet, where poorly solvated ions resulted in signal enhancement based on the concentration of electrolyte present [9]. Amad et al. have demonstrated the importance of gas-phase proton affinity in the response of positive ion electrospray ionization [11]. In negative ionization, in the case where the ion forms as a result of a loss of a proton (or loss of charge balancing cation, if present as a salt), the addition of fluorinated alcohols has been shown to enhance the signal of oligonucleotides, which has been attributed to volatility [15] and solution phase chemistry [16]. Huber and Krajete have shown the effects of volatile acids, bases, and solvents added via sheath flow on the response of oligonucleotides [17], where the addition isopropyl alcohol, methanol, and acetonitrile enhanced the analyte $\mathrm{S} / \mathrm{N}$.

We have undertaken studies in signal enhancement of ESI LC-MS/MS to have a practical method of reducing the limits of detection for the primary metabolites of organophosphorus nerve agents for response to chemical terrorism incidents. We report the effect of signal enhancement for methylphosphonic acids by postcolumn addition of aprotic solvents and longer chain alcohols. This response enhancement is accompanied by gains in the $\mathrm{S} / \mathrm{N}$ of the chromatographic peaks for these analytes, although to a lesser extent. A simple and generally applicable experimental setup is also discussed.

\section{Experimental}

\section{Materials}

The mixture of isotopically labeled analogues of the methylphosphonic acid metabolites $(500 \mu \mathrm{g} / \mathrm{L}$ in water) were purchased from Cerilliant Corporation (Round Rock, TX): EMPA (ethyl methylphosphonic acid, CAS 1832-53-7, ethyl- $\mathrm{D}_{5}$ ); IMPA (isopropyl methylphosphonic acid, CAS 1832-54-8, isopropyl $-{ }^{13} \mathrm{C}_{3}$ ); PMPA (pinacolyl methylphosphonic acid, CAS 616-52-4, trimethylpropyl- ${ }^{13} \mathrm{C}_{6}$ ); CMPA (cyclohexyl methylphosphonic acid, CAS 1932-60-1, cyclohexyl- $\left.-{ }^{13} \mathrm{C}_{6}\right)$; and MMPA (2-(methyl)propyl methylphosphonic acid, CAS 1604-38-2, methylphosphonyl- $\left.{ }^{13} \mathrm{C}, \mathrm{D}_{3}\right)$. The working solution was prepared by diluting $50 \mu \mathrm{L}$ of this mixture to $1 \mathrm{~mL}$ with acetonitrile.

Organic-free 18.2 M $\Omega$ Type I water from a purifier purchased from Aqua Solutions, Inc. (Jasper, GA) was used in these studies. Omnipur ammonium acetate $(97 \%)$ was purchased from EMD Chemical, Inc. (Gibbstown, NJ). HPLC grade acetonitrile, HPLC grade ethyl acetate, pesticide grade methanol, pesticide grade acetone, and certified grade 1-propanol were purchased from Fisher Scientific (Fairlawn, NJ). HPLC-grade 1,4dioxane and HPLC grade isopropyl alcohol was purchased from Acros Organics (Morris Plains, NJ). Chromosolv Plus HPLC grade $\mathrm{N}, \mathrm{N}$-dimethyl formamide and ACS spectrophotometric grade ethanol were purchased from Sigma Aldrich (St. Louis, MO).

\section{Instrumental Analysis}

An Agilent 1100 HPLC with a well-plate autosampler (Santa Clara, CA) was used in these studies. The HPLC column employed for the methylphosphonic acids was a Waters Atlantis HILIC $1-\mathrm{mm} \times 50-\mathrm{mm}$ with $3 \mu \mathrm{m}$ particles. Five $\mu \mathrm{L}$ of the working solution was injected onto the column. The mobile phase was composed of $86 \%$ acetonitrile and $14 \% 20 \mathrm{mM}$ ammonium acetate and pumped at a flow rate of $150 \mu \mathrm{L} / \mathrm{min}$. Post-column addition was performed with a separate Agilent 1100 binary pump operating at $280 \mu \mathrm{L} / \mathrm{min}$, plumbed into a three-way union on the mass spectrometer. When changing solvents, the system was purged in excess of 20 system volumes to ensure that there was no contamination from the previous solvent.

The mass spectral analysis was performed on an API 4000 triple quadrupole mass spectrometer from Applied Biosystems (Foster City, CA) controlled by Analyst software. The mass spectrometer ion source was operated in negative electrospray ionization mode, and either selected reaction monitoring (SRM) or Q1 full scan mode, depending on the type of experiment. The specific settings used were the same as those in [2].

\section{Data Analysis}

The data were analyzed using Analyst 1.4.1, which was provided with the instrument. This software allows review of the chromatograms, as well as the integration and signal-to-noise $(\mathrm{S} / \mathrm{N})$ calculation of the chromatographic peaks. Analyte response is defined as the integrated area of the respective chromatographic peak. The $\mathrm{S} / \mathrm{N}$ values for all the chromatograms were determined using the same program settings and the same noise region of 1.75 to $2.25 \mathrm{~min}$. All comparisons of S/N values are relative to the post- column addition of the mobile phase to take into account any effects related to flow rate. All S/N values are the average over four separate measurements.

\section{Results and Discussion}

\section{Experimental Setup}

Infusion experiments conducted during method development studies for methylphosphonic acids revealed that analyte response and signal-to-noise ratio $(\mathrm{S} / \mathrm{N})$ were enhanced with increasing acetonitrile content [2], especially at compositions above $80 \%$ acetonitrile. The plot of response and $\mathrm{S} / \mathrm{N}$ versus acetonitrile content revealed the largest gains occurred above $90 \%$ acetonitrile, maximizing near $95 \%$. Unfortunately, a chromatographic system could not be devised that produced acceptable chromatographic peak shape with such mobile phase compositions. In fact, acetonitrile concentrations at and above $95 \%$ resulted in complete retention of some of the compounds on the silica stationary phase. 


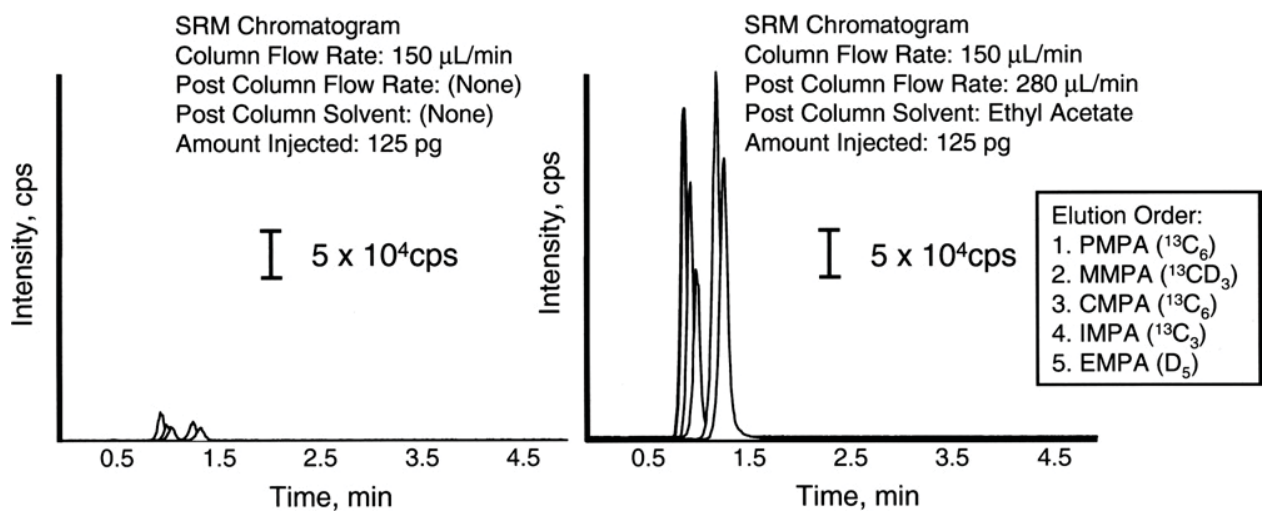

Figure 1. Comparison of SRM chromatograms resulting from no post-column addition and the post-column addition of ethyl acetate for the alkyl methylphosphonic acids. EMPA: ethyl $\left(\mathrm{D}_{5}\right)$ methylphosphonic acid; IMPA: isopropyl $\left({ }^{13} \mathrm{C}_{3}\right)$ methylphosphonic acid; MMPA: (2-methyl)propyl methyl $\left({ }^{13} \mathrm{CD}_{3}\right)$ phosphonic acid; CMPA: cyclohexyl $\left({ }^{13} \mathrm{C}_{6}\right)$ methylphosphonic acid; PMPA: pinacolyl $\left({ }^{13} \mathrm{C}_{6}\right)$ methylphosphonic acid.

One practical approach to obtain this solvent composition and insure adequate mixing before introduction to the ionization source was through post-column addition. This was accomplished via the stock threeway connector incorporated onto the ionization source of the mass spectrometer, with 18 inches of 0.007 " PEEK tubing joining this connector to the ESI needle assembly. Additional lengths of tubing did not enhance the observed effect, so this length was deemed to allow adequate mixing of the solvents. This setup dilutes the chromatographic peak by about 3 to 1 to achieve the desired concentration of organic solvent for these analytes. It was found that post-column addition of several organic solvents resulted in response and $\mathrm{S} / \mathrm{N}$ enhancement that greatly surpassed the resultant dilution of the analytes (Figure 1).

\section{Response Enhancement}

The increase in response of an analyte in ESI LCMS/MS can occur for a variety of reasons. Typically, this is due to an increase in the concentration of the analyte in the chromatographic peak, such as injecting increasing concentrations of analyte when building a quantitative calibration curve. However, response can also be increased by enhancing the generation of gasphase analyte ions. This can be accomplished by manipulating various ion source parameters or through changes in mobile phase composition and flow rate. In any case, the response is directly proportional to the number of analyte ions that are produced in the ionization source and subsequently sampled by the mass spectrometer. Therefore, any gains in the responses of the analytes observed in these experiments are the result of an increase in the number of analyte ions liberated into the gas phase, assuming the efficiency of ion transfer through the interface remains constant.

The post-column addition of organic solvents enhances the response of the methylphosphonic acids by negative ion ESI LC-MS/MS by up to a factor of 23 for
EMPA $\left(D_{5}\right)$ with ethyl acetate (Figure 1). Interestingly, the post-column addition dilutes the chromatographic peak by a factor of 3 to 1 , resulting in a lower concentration of the analyte. This means that the increase in response must be due to an increase in the delivery of the analyte ions into the gas-phase caused by the composition of the electrosprayed solution. Further, to investigate any effects that might arise from flow rate, the post-column addition of the mobile phase was also investigated. This was found to lower the response by a factor of 1.5 to 2.6 depending on the analyte (Table 1). This can be attributed to the dilution of the chromatographic peak and effects on the ESI process related to change in flow rate. All comparisons made from here on will be relative to the post-column addition of mobile phase to eliminate any flow rate and analyte concentration effects.

There are a number of physical properties related to the solvent composition that can enhance analyte response in electrospray ionization mass spectrometry. For flow rates typically encountered in LC-MS/MS analysis, the initial electrosprayed droplet is too large to generate a significant number of gas-phase ions. The droplet must decrease in size while maintaining its excess charge through the evaporation of neutral solvent to the point where offspring droplets are formed, which proceed to generate a significant number of gas-phase ions $[18,19]$. Therefore, increasing the rate of neutral solvent evaporation typically enhances the instrument response and is accomplished through the incorporation of more volatile organic solvents in the mobile phase. Interestingly, this effect did not occur conclusively in these studies. For instance, Table 2 shows that the response enhancement of EMPA was four times larger with $\mathrm{N}, \mathrm{N}^{\prime}$-dimethyl formamide $\left(\right.$ B.P. $=153^{\circ} \mathrm{C}$ ) compared with methanol (B.P. $=65^{\circ} \mathrm{C}$ ).

The tested organic solvents possess a lower surface tension and dielectric constant compared to water, which ${ }^{\circ}$ tend $^{\circ}$ to $^{\circ}$ lead $^{\circ}$ to $^{\circ}$ smaller $^{\circ}$ initial ${ }^{\circ}$ droplets $^{\circ}\left[20^{\circ}-22\right]$, increasing the efficiency of ionization and instrument 
Table 1. Effects of the post-column addition solvent on the response and signal to noise $(\mathrm{S} / \mathrm{N})$ of various analytes

\begin{tabular}{|c|c|c|c|c|c|c|c|c|c|c|}
\hline \multirow[b]{2}{*}{ Post-column addition solvent } & \multicolumn{5}{|c|}{$\begin{array}{l}\text { Response relative to post-column addition } \\
\text { of mobile phase }\end{array}$} & \multicolumn{5}{|c|}{$\begin{array}{l}\mathrm{S} / \mathrm{N} \text { relative to post-column addition } \\
\text { of mobile phase }\end{array}$} \\
\hline & EMPA & IMPA & MMPA & CMPA & PMPA & EMPA & IMPA & MMPA & CMPA & PMPA \\
\hline No. post-column & 2.6 & 2.3 & 2.1 & 2.3 & 2.1 & 1.5 & 2 & 1.6 & 2.2 & 1.6 \\
\hline Mobile phase & 1 & 1 & 1 & 1 & 1 & 1 & 1 & 1 & 1 & 1 \\
\hline $\mathrm{N}, \mathrm{N}$-dimethyl formamide & 7.7 & 6.3 & 4.5 & 3.3 & 3.6 & 1.8 & 2.2 & 1.5 & 3.1 & 1.9 \\
\hline Acetone & 8.8 & 7.5 & 6.2 & 6.2 & 5.4 & 3.3 & 3.3 & 2.4 & 3.9 & 2.2 \\
\hline 1.4-dioxane & 44 & 34.2 & 27.6 & 23.4 & 19.4 & 7.1 & 6.8 & 10.9 & 8.2 & 5.7 \\
\hline Acetonitrile & 47.9 & 36.3 & 28.4 & 25.5 & 19.8 & 10.9 & 9.9 & 19.1 & 14.4 & 9.2 \\
\hline Ethyl acetate & 60.3 & 46 & 35.2 & 30.2 & 24.6 & 11.2 & 9.2 & 13.4 & 12.4 & 7.7 \\
\hline Water & 0.9 & 1 & 1.1 & 1.2 & 1.1 & 0.7 & 0.9 & 0.9 & 1.1 & 0.9 \\
\hline Methanol & 1.6 & 1.7 & 1.8 & 1.8 & 1.9 & 0.7 & 1.4 & 1 & 2 & 1.5 \\
\hline Ethanol & 2 & 1.9 & 1.6 & 1.4 & 1.5 & 1.3 & 1.6 & 1 & 0.8 & 0.7 \\
\hline 2-Propanol & 18.3 & 14.6 & 11.4 & 9.3 & 8 & 5.6 & 4.8 & 3.2 & 5.5 & 3.3 \\
\hline 1-Propanol & 33.9 & 26.8 & 21.1 & 17.5 & 14.7 & 7.3 & 8.1 & 6.9 & 3.6 & 5.7 \\
\hline
\end{tabular}

All values are ratioed to the post-column addition of mobile phase in order rule out any effects of flow rate, and the maximum values are in bold. EMPA: ethyl $\left(D_{5}\right)$ methylphosphonic acid; IMPA: isopropyl $\left({ }^{13} \mathrm{C}_{3}\right)$ methylphosphonic acid; MMPA: (2-methyl)propyl methyl $\left({ }^{13} \mathrm{CD}_{3}\right)$ phosphonic acid, CMPA is cyclohexyl $\left({ }^{13} \mathrm{C}_{6}\right)$ methylphosphonic acid; PMPA: pinacolyl $\left({ }^{13} \mathrm{C}_{6}\right)$ methylphosphonic acid.

response. The data presented here do not follow a strict trend with respect to surface tension (compare ethyl ${ }^{\circ}$ acetate ${ }^{\circ}$ to $^{\circ}$ methanol ${ }^{\circ}$ in $^{\circ}$ Table $^{\circ} 2,{ }^{\circ}$ for ${ }^{\circ}$ instance $)^{\circ}$ or dielectric constant (comparing acetonitrile with 1,4dioxane ${ }^{\circ}$ and $^{\circ}$ ethy $^{\circ}{ }^{\circ}$ acetate $^{\circ}$ in $^{\circ}$ Table $^{\circ} 2$ )..$^{\circ}$ Last, ${ }^{\circ}$ since ${ }^{\circ}$ the analyte ions must form through the transfer of a proton (or other charge balancing cationic species) to another compound present in the droplet to become part of the excess charge, solvents with larger gasphase proton affinities may lead to an enhanced response. The response data collected, summarized in Table $^{\circ} 2{ }^{\circ}$ with $^{\circ}$ the $^{\circ}$ aprotic $^{\circ}$ solvents $^{\circ} \operatorname{did}^{\circ}$ not $^{\circ}$ show $^{\circ}$ a dependence $^{\circ}$ on $^{\circ}$ gas-phase $^{\circ}$ proton $^{\circ}$ affinity $^{\circ}$ as $^{\circ}$ in $^{\circ}[11]$. This may be due to the relatively large structural differences in these molecules and differences in chemical properties such as the relatively high boiling point of $\mathrm{N}, \mathrm{N}$-dimethylformamide.

Two general trends can be noted in the data. First, the post-column addition of protic solvents enhanced the response and $\mathrm{S} / \mathrm{N}$ in the order of: $\mathrm{H}_{2} \mathrm{O}<$ meth- anol $<$ ethanol $<$ 1-propanol $<$ 2-propanol. On average, the post-column addition of water, methanol, and ethanol resulted in little or no enhancement of the response. However, 1-propanol and 2-propanol resulted in an $\mathrm{S} / \mathrm{N}$ enhancement similar to several aprotic solvents, with the aprotic solvents resulting in the largest effect. One might expect methanol to form clusters with the analyte ions, resulting in the reduction of SRM intensity. When adding methanol postcolumn, there is no significant formation of clusters with EMPA as demonstrated in the full scan spectrum in Figure 2. We therefore propose that this trend reflects the ability of the smaller protic solvents to stabilize anion solvation in the droplets through hydrogen bonding interactions. This stabilization would obviously not occur in aprotic solvents and may explain the relatively large differences in enhancement between methanol and the aprotic solvents $^{\circ}$ in $^{\circ}$ Table $^{\circ} 2 .^{\circ}$ Otherwise, ${ }^{\circ}$ it $^{\circ}$ can $^{\circ}$ be $^{\circ}$ said $^{\circ}$ that solvents with lower boiling points, lower surface

Table 2. Comparison of the response signal enhancement with chemical properties that have been shown to effect analyte response in electrospray ionization

\begin{tabular}{|c|c|c|c|c|c|}
\hline $\begin{array}{c}\text { Post-column addition } \\
\text { solvent }\end{array}$ & $\begin{array}{l}\text { Effect on EMPA } \\
\text { response }\end{array}$ & $\begin{array}{c}\text { Gas-phase proton affinity } \\
\text { (kcal/mol) [23] }\end{array}$ & $\begin{array}{l}\text { Surface tension } \\
(\mathrm{mN} / \mathrm{m})[24]\end{array}$ & $\begin{array}{c}\text { Boiling } \\
\text { point }\left({ }^{\circ} \mathrm{C}\right) \\
{[24]}\end{array}$ & $\begin{array}{c}\text { Dielectric } \\
\text { constant }^{\circ}[24] \\
\end{array}$ \\
\hline \multicolumn{6}{|l|}{ Aprotic solvents } \\
\hline $\mathrm{N}, \mathrm{N}$-dimethylformamide & 7.7 & 212 & 35 & 153 & 38.3 \\
\hline Acetone & 8.8 & 194 & 23 & 56 & 21.0 \\
\hline 1,4-Dioxane & 44 & 190 & 33 & 101 & 2.2 \\
\hline Acetonitrile & 47.9 & 186 & 29 & 82 & 36.6 \\
\hline Ethyl acetate & 60.3 & 200 & 23 & 72 & 6.0 \\
\hline \multicolumn{6}{|l|}{ Protic Solvents } \\
\hline Water & 0.9 & 165 & 72 & 100 & 80.4 \\
\hline Methanol & 1.6 & 180 & 22 & 65 & 33.1 \\
\hline Ethanol & 2 & 186 & 22 & 78 & 25.3 \\
\hline Isopropyl alcohol & 18.3 & 190 & 23 & 82 & 20.1 \\
\hline 1-Propanol & 33.9 & 188 & 21 & 97 & 20.8 \\
\hline
\end{tabular}

EMPA: ethyl $\left(D_{5}\right)$ methylphosphonic acid. 


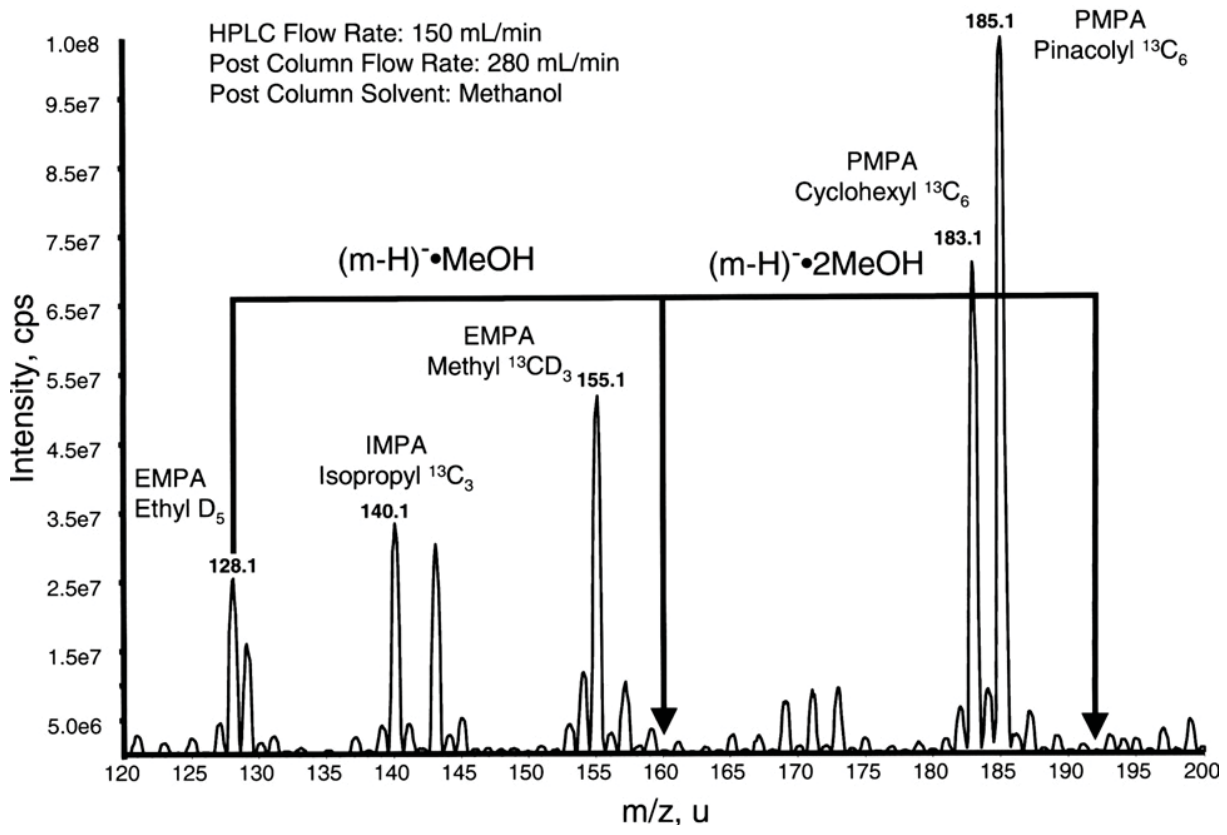

Figure 2. Full scan spectrum of the analytes demonstrating the lack of cluster formation with ethyl $\left(D_{5}\right)$ methyl phosphonic acid during the post-column addition of methanol.

tensions, and larger gas-phase proton affinities tended to increase the response for these analytes.

\section{Signal to Noise}

The main interest in this study was to find a simple method to obtain lower limits of detection (LODs) for the alkyl methylphosphonic acids. The key to lowering the LODs while keeping the sample size constant is through increasing the $\mathrm{S} / \mathrm{N}$ for the chromatographic peaks for the analytes of interest. Unfortunately, increases in detector response are not always met with increases in $\mathrm{S} / \mathrm{N}$ due to resultant increases in noise; otherwise decreasing the LOD for analyte would simply be a matter of increasing the detector gain.

The method presented here not only increases the response for the analytes, but also results in increases in the S/Noror the alkylmethyl'phosphonic ${ }^{\circ}$ acids'(Table 1 ). The maximum increase in $\mathrm{S} / \mathrm{N}$ obtained was for the

\section{Signal of MMPA SRM Chromatogram}

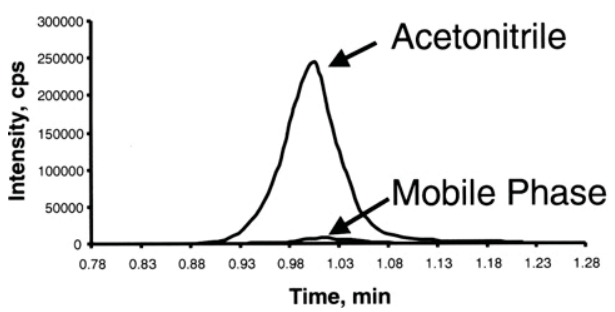

combination of MMPA $\left({ }^{13} \mathrm{CD}_{3}\right)$ and acetonitrile, which corresponds to the initial metabolite and hydrolysis product of rVX. A comparison of the analyte signal and noise areas used for this calculation are presented in Figure 3 , ${ }^{\circ}$ clearly ${ }^{\circ}$ showing ${ }^{\circ}$ the increase in ${ }^{\circ} \mathrm{S} / \mathrm{N}^{\circ}$ compared to the post-column addition of mobile phase. Interestingly, ethyl acetate gave the largest gains in response for all the analytes. However, while this solvent produced the largest $\mathrm{S} / \mathrm{N}$ increase with EMPA, acetonitrile produced the largest gains in $\mathrm{S} / \mathrm{N}$ for the remaining four analytes. This was due to a larger increase in baseline noise with ethyl acetate compared to acetonitrile for these analytes. The underlying cause for this discrepancy is not understood at this time.

\section{Conclusions}

The post-column addition of aprotic solvents and longer chain alcohols results in a significant increase of
Noise of MMPA SRM Chromatogram

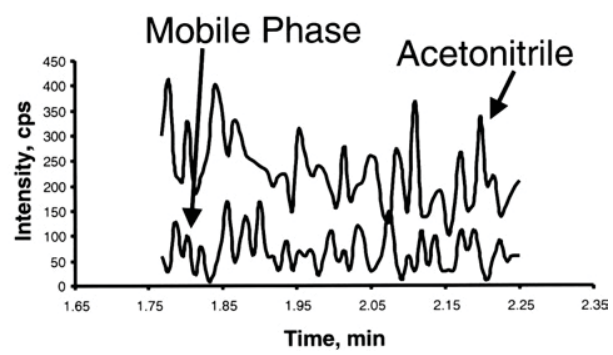

Figure 3. Comparison of signal and signal to noise $(S / N)$ regions of the SRM chromatogram of MMPA with the post-column addition of acetonitrile. MMPA: 2-methyl propyl methyl $\left({ }^{13} \mathrm{CD}_{3}\right)$ phosphonic acid. 
the response for alkyl methylphosphonic acids in negative ionization ESI LC-MS/MS. The increase in response represents an increase in the number of analyte ions introduced into the gas phase and sampled by the mass spectrometer. This effect was much lower for the post-column addition of water, methanol, and ethanol; it is proposed that this is due to the ability of these solvents to stabilize the deprotonated analyte solvation through hydrogen bonding. Although other chemical properties that are known to effect response do not follow a strict trend in these experiments, their effect cannot be disregarded. This is due in part to the relatively complex system of up to three solvents and background electrolyte present due to the chromatography. However, because the effect occurs in spite of this, it should be readily applicable to other chromatographic systems.

From a more practical standpoint, the $\mathrm{S} / \mathrm{N}$ of the chromatographic peak is fundamentally important to accurate LC-MS/MS measurements, and the increase in $\mathrm{S} / \mathrm{N}$ should represent a lowering of detection limits for this class of compounds. Further work will concentrate on investigating whether or not this effect carries over to lower the limits of detection in the LC-MS/MS analysis of biological and environmental samples where matrix effects are present. Since this effect is present with the alkyl methylphosphonic acids, which represent the initial metabolites and environmental degradation products of the corresponding organophosphorus nerve agents, it should be readily applicable to forensic, public health, and perhaps environmental assays where lower limits of detection are needed. Additionally, it would be interesting to investigate if this effect can be applied to a broader range of compounds analyzed by negative ionization ESI LC-MS/MS.

\section{References}

1. Newmark, J. Nerve Agents. Neurol. Clin. 2005, 23, 623-641.

2. Mawhinney, D. B., Hamelin, E. I., Fraser, R., Silva, S. S., Pavlopoulos, A. J., Kobelski, R. J. The Determination of Organophosphonate Nerve Agent Metabolites in Human Urine by Hydrophilic Interaction Liquid Chromatography Tandem Mass Spectrometry. J. Chromatogr. B, in press.
3. King, R.; Bonfiglio, R.; Fernandez-Metzler, C.; Miller-Stein, C.; Olah, T. Mechanistic Investigation of Ionization Suppression in Electrospray Ionization. J. Am. Soc. Mass Spectrom. 2000, 11, 942-950.

4. Schmidt, A.; Karas, M.; Dulcks, T. Effect of Different Solution Flow Rates on Analyte Ion Signals in Nano-ESI MS, or: When Does ESI Turn into Nano-ESI. J. Am. Soc. Mass Spectrom. 2003, 14, 492-500.

5. Beaudry, F.; Vachon, P. Electrospray Ionization Suppression, a Physical or a Chemical Phenomenon. Biomed. Chromatogr. 2006, 20, 200-205.

6. Weaver, R.; Riley, R. J. Identification and Reduction of Ion Suppression Effects on Pharmacokinetic Parameters by Polyethylene Glycol 400. Rapid Commun. Mass Spectrom. 2006, 20, 2559-2564.

7. Iavarone, A. T.; Udekwu, O. A.; Williams, E. R. Buffer Loading for Counteracting Metal Salt-Induced Signal Suppression in Electrospray Ionization. Anal. Chem. 2004, 76(14), 3944-3950.

8. Mallet, C. R.; Lu, Z.; Mazzeo, J. R. A Study of Ion Suppression Effects in Electrospray Ionization from Mobile Phase Additives and Solid-Phase extracts. Rapid Commun. Mass Spectrom. 2004, 18, 49-58.

9. Constantopoulos, T. L.; Jackson, G. S.; Enke, C. G. Effects of Salt Concentration on Analyte Response Using Electrospray Ionization Mass Spectrometry. J. Am. Soc. Mass Spectrom. 1999, 10, 625-634.

10. Hop, C. E. C. A.; Chen, Y.; Yu, L. J. Uniformity of Ionization Response of Structurally Diverse Analytes Using a Chip-Based nNanoelectrospray Ionization Source. Rapid Commun. Mass Spectrom. 2005, 19, 3139 3142 .

11. Amad, M. H.; Cech, N. B.; Jackson, G. S.; Enke, C. G. Importance of Gas-Phase Proton Affinities in Determining the Electrospray Ionization Response for Analytes and Solvents. J. Mass Spectrom. 2000, 35, 784-789.

12. Tang, L.; Kebarle, P. Effect of the Conductivity of the Electrosprayed Solution on the Electrospray Current. Factors Determining Analyte Sensitivity in Electrospray Mass Spectrometry. Anal. Chem. 1991, 63(23), 2709-2715.

13. Tang, L.; Kebarle, P. Dependence of Ion Intensity in Electrospray Mass Spectrometry on the Concentration of the Analytes in the Electrosprayed Solution. Anal. Chem. 1993, 65(24), 3654-3668.

14. Enke, C. G. A Predictive Model for Matrix and Analyte Effects in Electrospray Ionization of Singly-Charged Ionic Analytes. Anal. Chem. 1997, 69(23), 4885-4893.

15. Apffel, A.; Chakel, J. A.; Fischer, S.; Lichtenwalter, K.; Hancock, W. S. Analysis of Oligonucleotides by HPLC-Electrospray Ionization Mass Spectrometry. Anal. Chem. 1997, 69(7), 1320-1325.

16. Cech, N. B.; Enke, C. G. Practical Implications of Some Recent Studies in Electrospray Ionization Fundamentals. Mass Spectrom. Rev. 2001, 20, 362-387.

17. Huber, C. G.; Krajete, A. Sheath Liquid Effects in Capillary HighPerformance Liquid Chromatography-Electrospray Mass Spectrometry of Oligonucleotides. J. Chromatogr. A 2000, 870, 413-424.

18. Iribarne, J. V.; Thomson, B. A. On the Evaporation of Small Ions from Charged Droplets. J. Chem. Phys. 1976, 64(6), 2287-2294.

19. Kebarle, P.; Peschke, M. On the Mechanisms by which the Charged Droplets Produced by Electrospray Lead to Gas-Phase Ions. Anal. Chim. Acta 2000, 406, 11-35.

20. Rohner, T. C.; Lion, N.; Girault, H. H. Electrochemical and Theoretical Aspects of Electrospray Ionization. Phys. Chem. Chem. Phys. 2004, 6, 3056-3068.

21. Chen, D. R.; Pui, D. Y. H. Experimental Investigation of Scaling Laws for Electrospraying: Dielectric Constant effect. Aerosol Sci. Technol. 1997, 27(3), 367-380.

22. Smith, J. N.; Flagan, R. C.; Beauchamp, J. L. Droplet Evaporation and Discharge Dynamics in Electrospray Ionization. J. Phys. Chem. A 2002, 106(42), 9957-9967.

23. Aue, D. H.; Bowers, M. T. Gas Phase Ion Chemistry, Vol. II; Academic Press, Inc.: New York, 1979.

24. CRC Handbook of Chemistry and Physics, 85th ed.; CRC Press: New York, 2004. 